\title{
Chemical Kinetic Strategies for High-Throughput Screening of Protein Aggregation Modulators
}

Zsuzsa Sárkány, ${ }_{1}^{[d]}$ Fernando Rocha, ${ }^{[d]}$ Ana M. Damas, ${ }^{[c]}$ Sandra Macedo-Ribeiro, ${ }^{[a, b]}$ and Pedro M. Martins* $\left.{ }^{* a, b}, c\right]$

Kinetic Inhibitor


Abstract: Insoluble aggregates staining positive to amyloid dyes are known histological hallmarks of different neurodegenerative disorders and of type II diabetes. Soluble oligomers are smaller assemblies whose formation prior to or concomitant with amyloid deposition has been associated to the processes of disease propagation and cell death. While the pathogenic mechanisms are complex and differ from disease to disease, both types of aggregates are important biological targets subject to intense investigation in academia and industry. Here we review recent advances in the fundamental understanding of protein aggregation that can be used on the development of anti-amyloid and antioligomerization drugs. Specifically, we pinpoint the chemical kinetic aspects that should be attended during the development of high-throughput screening assays and in the hit validation phase. The strategies here devised are expected to establish a connection between basic research and pharmaceutical innovation.

\section{Introduction}

Over the recent years, protein aggregation has been in the limelight of drug discovery: on the one hand, several projects targeting amyloid aggregates in neurodegenerative disorders have been discontinued. ${ }^{[1]}$ On the other hand, smaller protein aggregates-the soluble oligomers-are increasingly surfacing as major culprits of neurotoxicity and pathogenic spread. ${ }^{[2]} \mathrm{Co}$ incidently with this paradigm shift, ${ }^{[3]}$ encouraging results have been obtained by the first generation of anti-oligomerization drug candidates in clinical trials of Alzheimer's disease and Parkinson's disease. ${ }^{[4]}$

The long and costly workflow leading to the discovery of new drugs has in its basis methods of high-throughput screening (HTS) of active compounds (known as "hits") that can be further developed into drug precursors (known as "leads") or used as "chemical probes" to validate a given biological target. ${ }^{[5]}$ Targeted HTS approaches rely on in vitro protein aggregation assays having well-defined reaction components and high reproducibility indexes. ${ }^{[6]}$ However, deciphering the exact mode of action of aggregation inhibitors is not a trivial task on account of the possible pathways (Figure $1 \mathrm{a}$ ) and multiple elementary steps (Figure $1 \mathrm{~b}$ ) that might be implicated. It may occur, for instance, that large amounts of stable soluble oligomers are formed without their presence being directly detect-

[a] Dr. S. Macedo-Ribeiro, Prof. P. M. Martins

IBMC-Instituto de Biologia Molecular e Celular

Universidade do Porto

4200-135 Porto (Portugal)

E-mail:pmartins@ibmc.up.pt

[b] Dr. S. Macedo-Ribeiro, Prof. P. M. Martins

Instituto de Investigação e Inovação em Saúde

Universidade do Porto

4200-135 Porto (Portugal)

[c] Prof. A. M. Damas, Prof. P. M. Martins

ICBAS-Instituto de Ciências Biomédicas Abel Salazar

Universidade do Porto

4050-313 Porto (Portugal)

[d] Dr. Z. Sárkány, Prof. F. Rocha

LEPABE-Departamento de Engenharia Química

Faculdade de Engenharia da Universidade do Porto

Rua Dr. Roberto Frias, 4200-465 Porto (Portugal)

(iD The ORCID identification number(s) for the author(s) of this article can be found under:

https://doi.org/10.1002/asia.201801703.

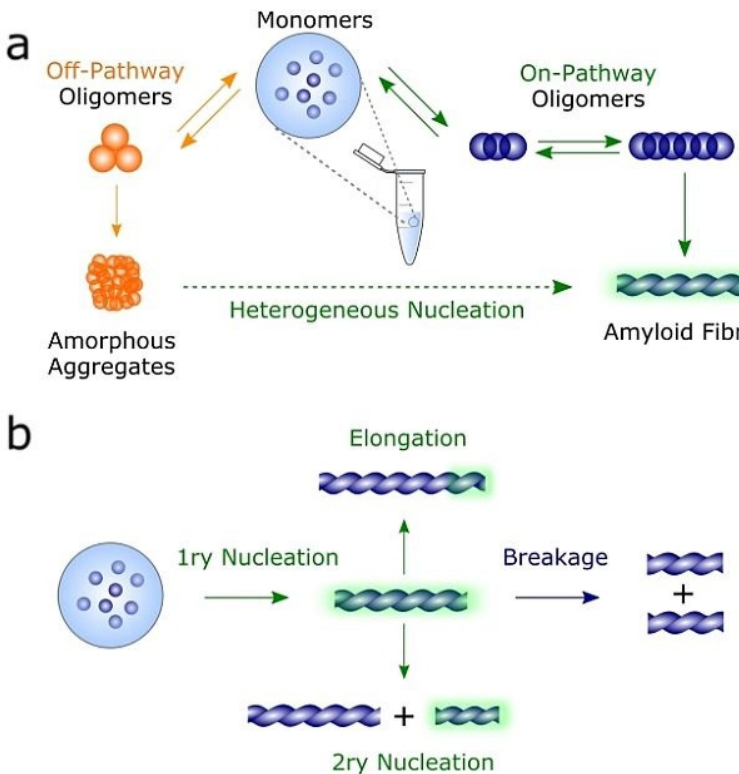

Figure 1. Aggregation pathways during amyloid fibril formation. a) Protein monomers establish a thermodynamic equilibrium with soluble oligomers that are either on- or off-pathway to produce amyloid fibrils. Off-pathway oligomers can undergo phase transition processes leading to the formation of different types of precipitates, ${ }^{[8]}$ and then act as heterogeneous nucleants of amyloid fibrils. b) Reaction steps participating in the amyloid pathway. Green glows represent an increase in the mass of amyloid fibrils. ${ }^{[9]}$ ed by amyloid-specific probes such as thioflavin-T (ThT) or by turbidimetric methods. ${ }^{[7]}$

Widely applied in the study of amyloid aggregation mechanisms, ${ }^{[10]}$ chemical kinetic analysis is gradually being established as a promising tool for the systematic screenings of both anti-amyloid and anti-oligomerization compounds. ${ }^{[1]}$ An important step in this direction was taken by defining the reference kinetic behavior expected for generic nucleation-andgrowth processes of phase transition. ${ }^{[12]}$ When no other pathway is present, the formation of insoluble species begins with the elementary step of primary nucleation and then proceeds through the occurrence of the autocatalytic steps of secondary nucleation and growth. Thermodynamic equilibrium is attained when the chemical potential of the solution equals that of the insoluble fraction. At that point, the concentration of protein monomer $(C)$ corresponds to the protein solubility $\left(C^{*}\right)$, and so the driving force for phase transition represented by supersa- 
turation $\Delta C=C-C^{*}$ (or in dimensionless form $\sigma=\Delta C / C^{*}$ ) is zero. After expressing supersaturation in terms of the normalized reaction conversion $(\alpha)$,

$\sigma=\sigma_{0}(1-\alpha)$

The following closed-form solution of the mass balance equations is obtained for unseeded reactions $\left(\alpha_{0}=0\right):{ }^{[12]}$

$\alpha=1-\frac{1}{k_{\mathrm{b}}\left[\exp \left(k_{\mathrm{a}} t\right)-1\right]+1}$

where $k_{\mathrm{a}}$ and $k_{\mathrm{b}}$ are determined by the elementary steps that increase the mass of insoluble aggregates (Figure $1 \mathrm{~b}$ ). Sigmoidal progress curves comprising an initial lag phase followed by an exponential phase and a plateau phase are expected in the cases of slow primary nucleation, ${ }^{[6,13]}$ for which the values of $k_{\mathrm{b}}$ are lower than $\sim 0.01 .^{[7,12]}$ In turn, fast primary nucleation processes $\left(k_{\mathrm{b}}>0.01\right)$ give rise to hyperbolic progress curves that are also observed during seeded aggregation assays (see section 2.). Protein aggregation modulators can be screened based on kinetic signatures standing out from the standard behavior expected by Equation (2). ${ }^{[7]}$ This is valid for both onand off-pathway inhibition as it is shown next for the case of amyloid fibril formation.

\section{The Amyloid Aggregation Assay}

Amyloid fibrils have a characteristic cross-beta-sheet structure that is detectable upon binding to Congo red, ThT and thiofla-

Zsuzsa Sárkány obtained her Ph.D. degree in Structural Biochemistry in 2006 from Eötvös Lóránd Unversity, Budapest, Hungary under the supervision of Prof. Dr. László Polgár. From 2007 to 2017 she worked at Institute for Molecular and Cell Biology (IBMC), Porto, Portugal with Prof. Dr. Ana Margarida Damas and Dr. Sandra Macedo-Ribeiro. She is currently a postdoctoral fellow at the Department of Chemical Engineering, University of Porto, Portugal with a research focus on exploring the oligomeric pathway of alpha synuclein protein through high-throughput screenings of drug-like compounds.

Fernando Rocha completed his Ph.D. degree in 1984 at the University of Porto. Since then he has worked as Assistant Professor in the Chemical Engineering Department of the University of Porto. His research interests include crystallization of biologically relevant molecules, with applications to health issues.
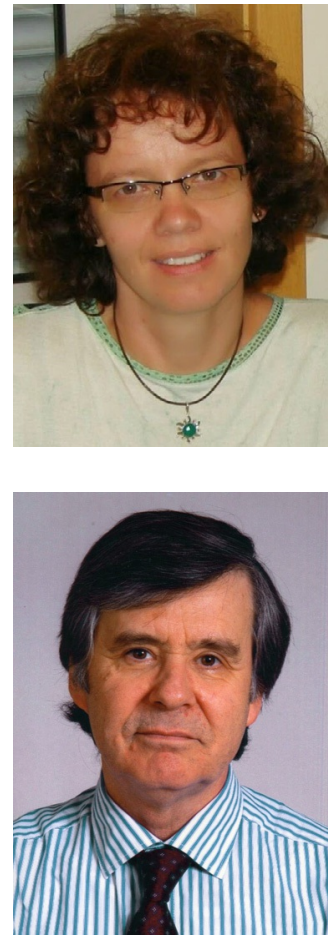

vin-S (ThS) dyes. ${ }^{[11 a, 14]}$ Although amyloid aggregation can be followed by other spectrophotometric techniques, ${ }^{[15]}$ thioflavin fluorescence is adequate for HTS kinetic assays that require low sample volume, high signal-to-noise ratios and high reproducibility. ${ }^{[6,11 b]}$ The use of this probe is not without pitfalls arising from non-amyloid binding, reduced sensibility to early (onpathway) aggregates and fluorescence self-quenching. ${ }^{[16]}$ As with other assays employing light-based detection methods, orthogonal validation using different output reporters is required in order to identify false positives and fluorescence artifacts. ${ }^{[17]}$ The development and optimization of HTS assays aims at rightfully interrogate a given therapeutic target using miniaturized formats of 96-well, 384-well or 1536-well microplates. ${ }^{[18]}$ Since amyloid nucleation is a stochastic process, ${ }^{[19]}$ some variability between replicate experiments is expectable even when homogeneous, highly pure protein samples are used. ${ }^{[20]}$ This is detrimental for the quality of the HTS assay usually characterized in terms of the $Z^{\prime}$ factor (Figure $2 \mathrm{a}$ ) taking into account

Ana M. Damas is a retired Full Professor of Biophysics of the Abel Salazar Biomedical School of the University of Porto. Her main research interests are X-ray Crystallography and Amyloidosis.

Sandra Macedo-Ribeiro completed her Ph.D. degree in 1999 at the Technical University of Munich. She then moved to the Institute for Molecular Biology in Barcelona as a postdoctoral researcher. She was Assistant Professor at the University of Algarve and Assistant Researcher at the Center for Neuroscience and Cell Biology in Coimbra. Presently, she is a Principal Researcher at the Institute for Molecular and Cellular Biology/Institute for Research and Innovation in Health (IBMC/i3S, Porto) and leader of the Biomolecular Structure and Function group. Her research interests include the structural and functional analysis of polyglutamine-rich proteins.

Pedro Martins completed his Ph.D. degree in Chemical Engineering with a Thesis on the physics of crystal growth (2006, University of Porto). He is a Research Assistant at the Biomolecular Structure and Function group of the Institute for Molecular and Cell Biology/Institute for Research and Innovation in Health (IBMC/i3S). His research interests include protein aggregation, enzymology and drug discovery.
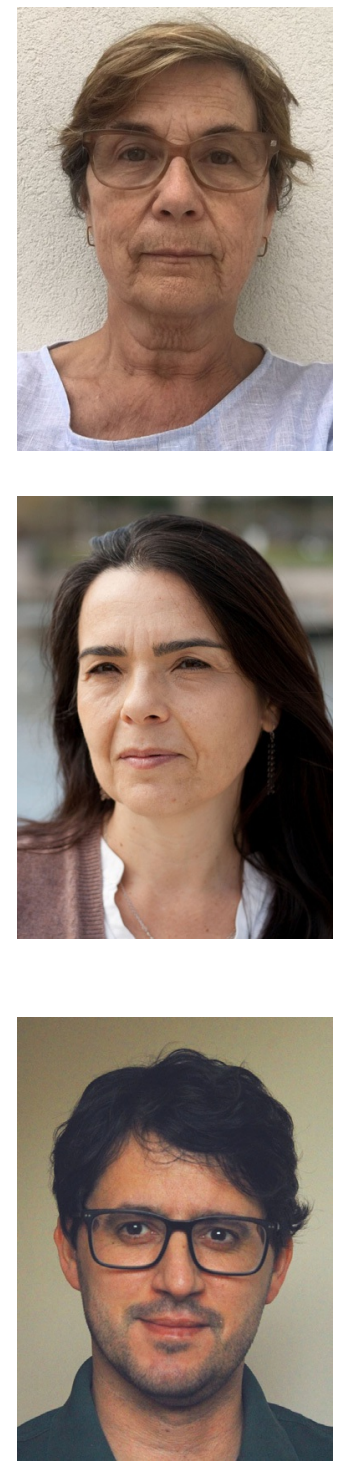

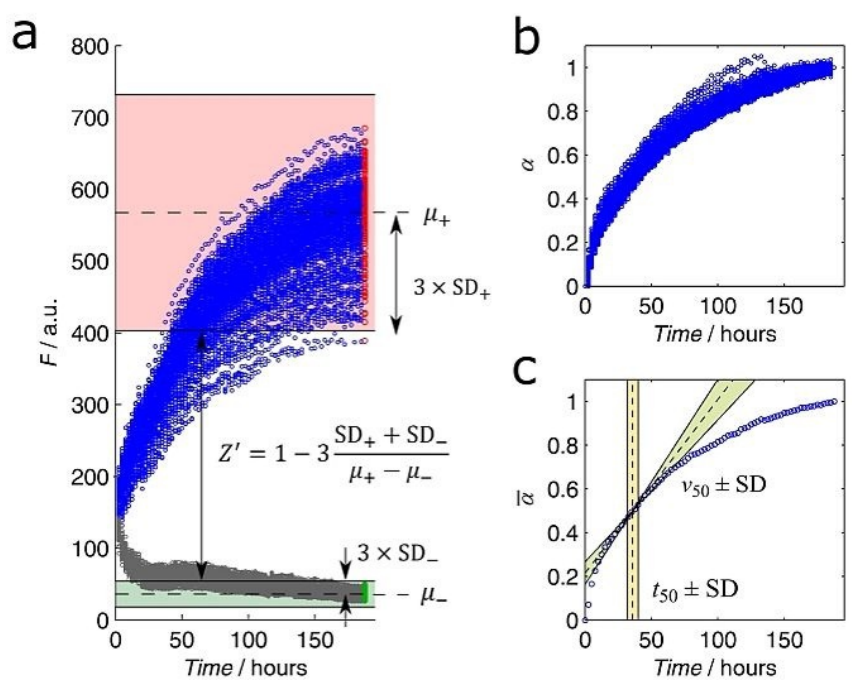

Figure 2. Example showing an optimized $\alpha$-synuclein aggregation assay. A good separation between positive and negative controls was achieved by adding $0.2 \mu \mathrm{M}$ of pre-formed fibrils (or "seeds") to the reaction mixture consisting of $2 \mu \mathrm{M} \alpha$-synuclein in $50 \mathrm{~mm}$ sodium phosphate $\mathrm{pH} 7.5,200 \mathrm{~mm}$ $\mathrm{NaCl}$ and $70 \mu \mathrm{M}$ ThT (Z. Sárkány and P. M. Martins, personal communication). a) Symbols: ThT fluorescence (F) measured over time in a 384-well plate, each well containing $0.2 \mu \mathrm{M}$ of seeds in the presence (blue) and absence (gray) of soluble $\alpha$-synuclein. A $Z^{\prime}$ factor of 0.66 was obtained using mean values ( $\mu$, dashed lines) and standard deviations (SD, shaded areas correspond to $3 \times \mathrm{SD}$ ) calculated for the $\mathrm{F}$ measurements at time point 187 hours $\left(F_{\mathrm{F}}\right)$ in 192 positive controls (red symbols) and 192 negative controls (green symbols). b) Amyloid conversion calculated as the normalized fluorescence increase $\left(\alpha=\Delta F / \Delta F_{\mathrm{F}}\right)$ taking as reference the instantaneous negative controls $\left(\Delta F=F-\mu_{-}\right)$. c) Mean values of amyloid conversion ( $\bar{\alpha}$, symbols), $t_{50}$ (vertical dashed line) and $v_{50}$ (sloping dashed line) calculated from the experimental data in (b). Shaded areas represent $\mu \pm$ SD intervals.

the means $(\mu)$ and standard deviations (SD) of positive and negative controls. A $Z^{\prime}$ factor $>0.5$ indicates a good degree of separation between controls. ${ }^{[21]}$ To improve reproducibility, pre-formed fibrils (or "seeds") can be added to the initial solution to bypass the primary nucleation step. ${ }^{[22]}$ The drawback of seeded assays is their insensibility to aggregation inhibitors specifically targeting non-templated nucleation. Other alternatives designed to accelerate fibrillation through the use of glass beads, vigorous agitation, high temperature, low $\mathrm{pH}$ and/ or denaturating additives only remotely reproduce the biological medium. ${ }^{[22]}$ Heterogeneous nucleants such as lipid micelles, can be used to mimic the cell membrane and disease-propagating exosomes, ${ }^{[23]}$ and at the same time catalyze protein aggregation with improved reproducibility. ${ }^{[24]}$ In principle, seeding is not expected to influence the ThT fluorescence increase $\left(\Delta F_{\mathrm{F}}\right)$, which is an important thermodynamic measurable (Figure $2 \mathrm{a}$ ) proportional to the initial supersaturation $\Delta C_{0}=C_{0}-C^{*}$. The kinetic influence of seeding is accounted by analyzing the normalized progress curves (Figure $2 \mathrm{~b}$ ) and the half-life coordinates $t_{50}$ and $v_{50}$ (Figure $2 \mathrm{c}$ ). The fundamental definitions of $t_{50}$ and $v_{50}$ are expressed in terms of apparent rate constants $k_{a}^{\prime}$ and $k_{b{ }^{\prime}}^{\prime}{ }^{[12]}$

$t_{50}=\frac{1}{k_{a}^{\prime}} \ln \left(\frac{1}{k_{\mathrm{b}}^{\prime}}+1\right)$ $v_{50}=\frac{k_{a}^{\prime}}{4}\left(k_{\mathrm{b}}^{\prime}+1\right)$

which in turn are influenced by the initial fraction of seeds $\alpha_{0}$ (computed in relation to the total polymerizable protein, $\Delta C_{0}$ ):

$$
\begin{aligned}
& k_{\mathrm{a}}^{\prime}=k_{a}\left(1+\alpha_{0}\right) \\
& k_{\mathrm{b}}^{\prime}=\frac{k_{\mathrm{b}}+\alpha_{0}}{1+\alpha_{0}}
\end{aligned}
$$

For unseeded reactions $\left(\alpha_{0}=0\right), k_{\mathrm{a}}^{\prime}=k_{\mathrm{a}}$ and $k_{\mathrm{b}}^{\prime}=k_{\mathrm{b}}$. As it follows from eq 2 , otherwise sigmoidal progress curves $\left(k_{\mathrm{b}}<0.01\right)$ will develop hyperbolic shapes if $>1 \%$ of preformed fibrils are added to the initial solution $\left(k_{\mathrm{b}}^{\prime}>0.01\right)$.

\section{Recognizable Modulators}

Timely reviews on protein aggregation modulation were put forward by for example, Doig and Derreumaux, ${ }^{[25]}$ Eisele et al., ${ }^{[26]}$ and Velander et al., ${ }^{[27]}$ where different classifications of inhibitors are adopted based on mechanistic and physicochemical considerations. Here we make a shorter selection of examples that are representative of the modes of action summarized in Figure 3. For the sake of simplicity each mechanism is illustrated separately, although synergistic and antagonistic effects may take place simultaneously. The selected examples are listed in Table 1.

Purely kinetic inhibitors (Figure $3 a$ ) slow down the rate of fluorescence increase without changing the final fluorescence, which is a value indicative of the amount of amyloid fibrils produced. This is achieved through kinetic inhibition of any of the steps of primary nucleation, secondary nucleation or fibril elongation that increase the amyloid-specific signal (indicated by green arrows in Figures 1 and 3). Fluorination of the hydrophobic residues valine or phenylalanine of the LVFFD peptide increases the duration of the lag phase of $A \beta 42$ aggregation without significantly changing the normalized aggregation rate $v_{50}$ or the value of the total fluorescence increase $F_{\infty}{ }^{[28]}$ Therefore, the fluorinated peptides are considered kinetic inhibitors acting upon the step of primary nucleation. ${ }^{[28]}$

The lead compound CLR01 is a molecular tweezer that specifically binds to lysine residues thus disrupting important interactions for protein aggregation. ${ }^{[29]}$ The impact of CLR01 on ThT progress curves changes according to the amyloidogenic protein being studied; in the case of $A \beta 40$ aggregation, a tenfold excess added during the exponential and plateau phases induced an evident disaggregating effect manifested by a progressive loss of amyloid signal. ${ }^{[30]}$ This trend, which has also been observed during incubation of $A \beta 42$ with brazilin, ${ }^{[31]}$ corresponds to the kinetic signature of fibril disaggregators (Figure $3 b$ ).

By preventing fragmentation, fibril stabilization (Figure $3 \mathrm{c}$ ) might reduce prion-like spreading of small aggregates within and between cells, as demonstrated for seeded spreading of $A \beta$ and tau using animal models of Alzheimer's disease. ${ }^{[26,32]}$ Tight binding fibril stabilizers might also decrease the amyloid 


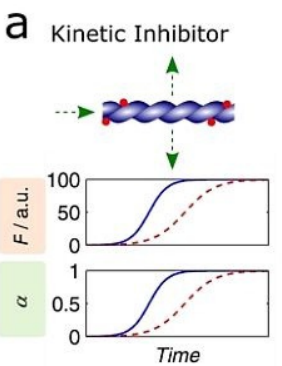

b

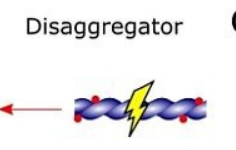

C

Fibril Stabilizer
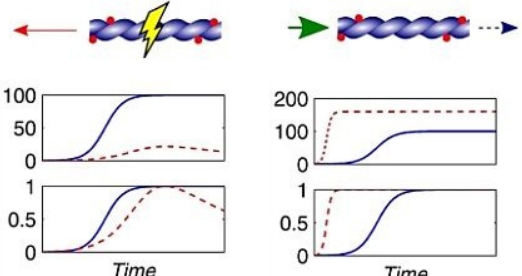

d Monomer Stabilizer

e

Oligomer Binder
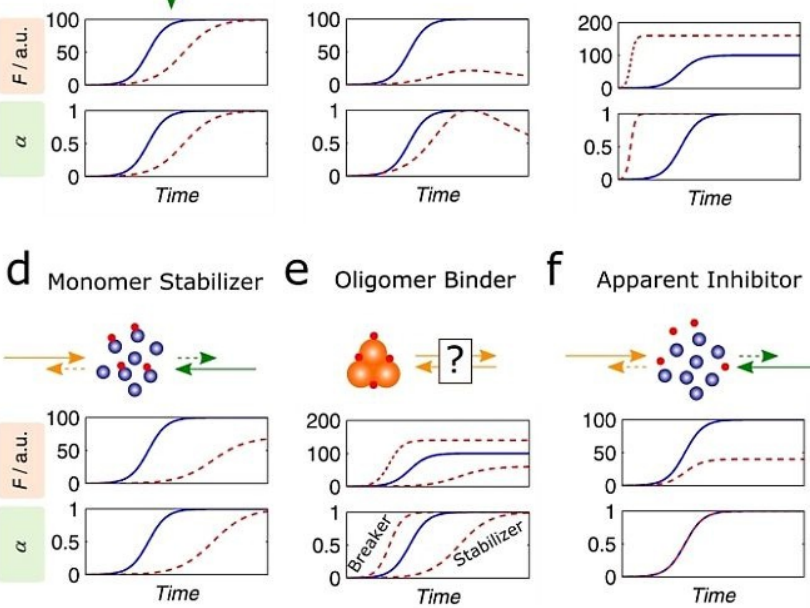

Time

$f$

Apparent Inhibitor

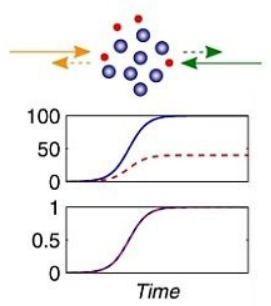

Figure 3. The different types of aggregation modulators recognizable by the amyloid aggregation assay. Each panel gives the mode of action (top, cartoon symbols as in Figure 1) and the kinetic signatures (middle and bottom) of each type of modulator; the progress curves represent the fluorescence (F) increase (middle) and the amyloid conversion (bottom) in the absence (solid lines) and presence (dashed lines) of test compound. The theoretical curves were computed using Equation (2) and assuming (i) $k_{\mathrm{a}}$ and $k_{\mathrm{b}}$ proportional to $\Delta C_{0} / C^{*}$, and (ii) $F$ proportional to the product $\Delta C_{0} \alpha e^{[9,43]}$ a) Kinetic inhibitors (associated to lower $k_{\mathrm{a}}$ and $k_{\mathrm{b}}$ values) decrease the rates of primary nucleation, secondary nucleation or fibril elongation (dashed arrows in the cartoon). b) Disaggregators provoke the dissociation of fibrils into soluble protein (red arrow in the cartoon). c) Fibril stabilizers decrease the values of solubility $\left(C^{*}\right)$ through stabilization of amyloid fibrils (large green arrow) and might prevent fibril breakage (dashed blue arrow) d) Monomer stabilizers decrease the aggregation propensity (dashed orange and green arrows) by inducing higher values of $C^{*}$. e) Oligomer binders may either stabilize or inhibit the formation of off-pathway oligomers, thereby decreasing or increasing the effective monomer concentration and the corresponding supersaturation value. f) Apparent inhibitors non-specifically alter the solution properties, which may reduce the thermodynamic propensity for aggregation (by reducing $\Delta C_{0}$ ) but do not affect $k_{\mathrm{a}}$ and $k_{\mathrm{b}}$ (as $\Delta C_{0} / C^{*}$ remains constant). ${ }^{[42]}$

solubility and redirect the equilibrium from potentially more toxic, small oligomers to amyloid fibrils (large green arrow in Figure $3 c) .[7,8,12]$ This hypothesis has been explored through the development of orange G-related compounds bound to the steric zipper $A \beta 16-21$ structure. $^{[33]}$

According to the supersaturation-dependence of $k_{\mathrm{a}}$ and $k_{\mathrm{b}}$, protein-specific modulation of solubility $\left(C^{*}\right)$ is an attractive approach to change the kinetics-and not only the thermodynamics - of amyloid fibril formation. This can be achieved using as target the amyloid pharmacophore of the soluble protein (Figure $3 \mathrm{c}$ ) or, alternatively, the metastable oligomers (Figures $3 \mathrm{~d}, \mathrm{e}$ ). Monomer stabilizers (Figure $3 \mathrm{~d}$ ) selectively increase the solubility of the target protein, shifting the on- and offpathway equilibria towards the dissociation of oligomers. As the result of the higher $C^{*}$ values, less amyloid fibrils are produced and lower fluorescence signals are expected at the end of the assay (Figure $3 \mathrm{~d}$, middle); because the values of supersaturation decrease, larger $t_{50}$ values and lower $v_{50}$ values are also expectable (Figure $3 d$, bottom). Monomer stabilizers and oligomer binders (Figure $3 \mathrm{e}$ ) are not clearly distinguishable from simple progress curves analysis if the mode of action of the latter type of inhibitor comprises the stabilization of the oligomeric assembly. This is because as the amyloidogenic protein is sequestered into the oligomeric state, the pool of available monomers is depleted leading to the train of events that culminates in lower $\Delta F_{\infty}$ values and slower aggregation kinetics. Mimics of the islet amyloid polypeptide (IAPP) synthesized with $\mathrm{N}$-methylated amide bonds were shown to inhibit the aggregation of IAPP and A $\beta 40$ by a common mechanism of stabilization of protein monomers and nontoxic oligomers. ${ }^{[34]}$ Accordingly, the IAPP mimics are included in the categories of protein and oligomer stabilizers (Figures $3 \mathrm{~d}, \mathrm{e}$, respectively). The stabilization of the transthyretin (TTR) tetramer by tafamidis (Vyndaqel; Pfizer), ${ }^{[35]}$ and by the repurposed drugs diflunis$\mathrm{al}^{[36]}$ and tolcapone ${ }^{[37]}$ inhibits the formation of amyloid deposits by preventing the dissociation of the tetramer into aggregation competent TTR monomers. Commonly referred as "kinetic stabilizers', ${ }^{\prime 26]}$ these compounds are here classified as oligomer stabilizers (Figure $3 \mathrm{e}$ ) to avoid confusion with the kinetic inhibitors having no thermodynamic effects on aggregation (Figure $3 a$ ).

The new evidences associating non-amyloidogenic oligomers to the pathogenesis of neurodegenerative diseases allow us to anticipate a renewed interest in oligomer breakers (Figure 3 e) as a therapeutic hypothesis to remodel toxic assemblies into nontoxic species. In the example of Figure $3 \mathrm{e}$, the aggregation equilibrium is redirected to the formation of more amyloid fibrils (Figure $3 \mathrm{e}$, top) and at faster rates (Figure $3 \mathrm{e}$, bottom). Other scenarios are, however, possible in which the amyloid pathway is not favored either because protein solubility is concomitantly increased or, as in the case of A 342 aggregation in the presence of the $\mathrm{N}$-methylated peptide inhibitor SEN304, ${ }^{[38]}$ other nontoxic pathways are elicited.

Table 1. Examples taken from the literature illustrating the different types of aggregation modulators presented in Figure 3.

\begin{tabular}{|c|c|}
\hline Modulator & Representative Examples \\
\hline Kinetic Inhibitor & - Peptide LVFFD with fluorinated valine or phenylalanine during A $\beta 42$ aggregation. ${ }^{[28]}$ \\
\hline Disaggregator & $\begin{array}{l}\text { - CLR01 during } A \beta 40 \text { aggregation. }{ }^{[30]} \\
\text { - Brazilin during } A \beta 42 \text { aggregation. }{ }^{[31]}\end{array}$ \\
\hline Fibril Stabilizer & - Orange G-related compounds bound to the steric zipper $A \beta 16-21$ structure. $^{[33]}$ \\
\hline Monomer Stabilizer & - Mimics of IAPP synthesized with $N$-methylated amide bonds during the aggregation of IAPP and A $\beta 40 .{ }^{[34]}$ \\
\hline Oligomer Binder & $\begin{array}{l}\text { - Tafamidis, }{ }^{[35]} \text { diflunisal }^{[36]} \text { and tolcapone }{ }^{[37]} \text { during the aggregation of TTR. } \\
\text { - N-Methylated peptide inhibitor SEN304 during the aggregation of A } \beta 422^{[38]}\end{array}$ \\
\hline Apparent Inhibitor & - Thermodynamic effects provoked by interfaces or by the addition of salts during amyloid aggregation. ${ }^{[42]}$ \\
\hline
\end{tabular}


Changing the ionic strength of amyloidogenic solutions by the addition (or removal) of salts can have a dramatic influence on amyloid aggregation in vitro, ${ }^{[39]}$ which is understood in terms of the role of solubility in determining the rate and extent of protein self-assembly. ${ }^{[7,8,12,40]}$ These modulation effects are considered "apparent" (Figure $3 \mathrm{f}$ ) since they are unspecific and tend to be buffered in cells and tissues media. Apparent inhibitors that solely alter the activity coefficient of the protein will originate superimposed progress curves if represented in normalized ThT fluorescence units (Figure $3 \mathrm{f}$, bottom); this has been previously illustrated using published data of Pronchik et al. ${ }^{[41]}$ on the influence of hydrophobic interfaces on $\alpha$-synuclein aggregation. ${ }^{[42]}$ It should be noted that the superimposition of normalized curves does not necessarily imply that the test compound should be discarded. In fact, Figure 3 does not exhaustively cover all possible mechanisms of inhibition, but instead it seeks to summarize the major types of modulators that can be identified by kinetic assays.

\section{Primary Screenings, Secondary Screenings, Hit Validation}

Having set up a reproducible amyloid aggregation assay does not guarantee that large numbers $\left(>10^{5}\right)$ of chemical compounds can be efficiently assessed during HTS campaigns, especially if several days are required to measure full progress curves. In these cases, a primary screening strategy can be devised to limit the measurement frequency to the initial, middle, and final points of the reaction (Figure 4). The corresponding values $F_{0}, F_{50}$ and $F_{F}$ (Figure $4 a$ ) can be followed in multiple plates simultaneously without advanced robotic systems being necessarily required. Positive hits stand out by showing $\Delta F_{50} / \Delta F_{F}$ ratios significantly different from the value of 0.5 expected in the absence of modulation effects and for apparent inhibitors (Figure $4 \mathrm{~b}$ ).

The 3-point aggregation assay can be used on the identification of positive hits as well as on the preliminary characterization of the putative inhibition mechanism. This requires the

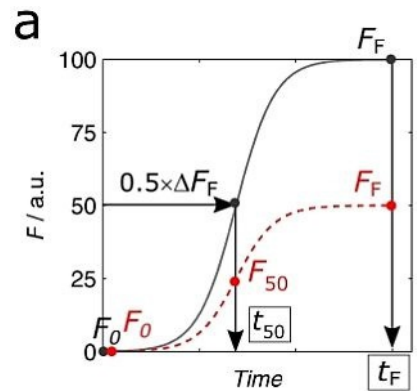

b

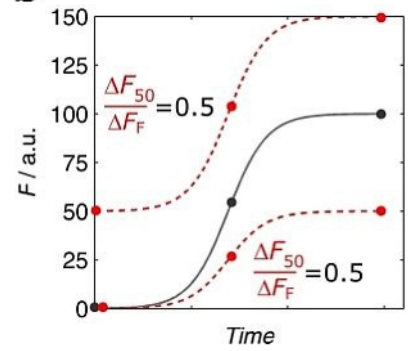

Figure 4. A possible strategy for primary screenings based on initial, midpoint and endpoint measurements. a) The midpoint $\left(t_{50}\right)$ and endpoint $\left(t_{\mathrm{F}}\right)$ instants are defined beforehand taking into account the control progress curve (solid line) characterizing the amyloid aggregation assay. Rather than continuously measuring the full progress curve in the presence of test compounds (dashed line), only the fluorescence increase at time-points $t_{50}$ $\left(\Delta F_{50}=F_{50}-F_{0}\right)$ and $t_{\mathrm{F}}\left(\Delta F_{\mathrm{F}}=F_{\mathrm{F}}-F_{0}\right)$, respectively, are measured. b) Negative results elicited by, e.g., fluorogenic compounds and apparent inhibitors can be identified by values of the $\Delta F_{50} / \Delta F_{F}$ ratio close to 0.5 . analysis of the $\Delta F_{50} / \Delta F_{F}$ ratio (Figure 5 a) but also of $\Delta F_{F}$ values obtained in the presence and absence of the test compound (Figure $5 \mathrm{~b}$ ). Kinetic inhibitors, for example, are expected to originate values of $\Delta F_{50} / \Delta F_{\mathrm{F}} \ll 0.5$ with no major impact on the values of $\Delta F_{F}$ (Figure $5 \mathrm{c}$ ). The progress curves characteristic of monomer and soluble oligomer stabilizers are not easily distinguishable (see previous representations in Figures $3 \mathrm{~d}$ and $3 \mathrm{e}$ ), and both are associated to markedly low values of $\Delta F_{50} / \Delta F_{F}$ and of $\Delta F_{F}$ (Figure $5 \mathrm{~d}$ ). Conversely, very high values of $\Delta F_{50} / \Delta F_{F}$ and of $\Delta F_{F}$ may indicate the cases of fibril stabilizers and of oligomer breakers (Figure $5 \mathrm{e}$ ).

In the same way that Figure 3 did not cover all possible inhibition mechanisms, Figure 5 gives a limited number of examples that can be identified by the 3-point screening assay. As expectable, this initial analysis is not free from ambiguities as exemplified in Figure 5 by cell B3, whose result can be ascribed

a

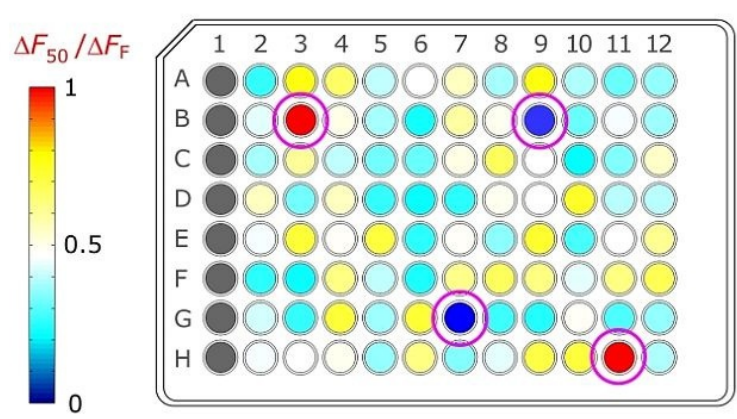

b

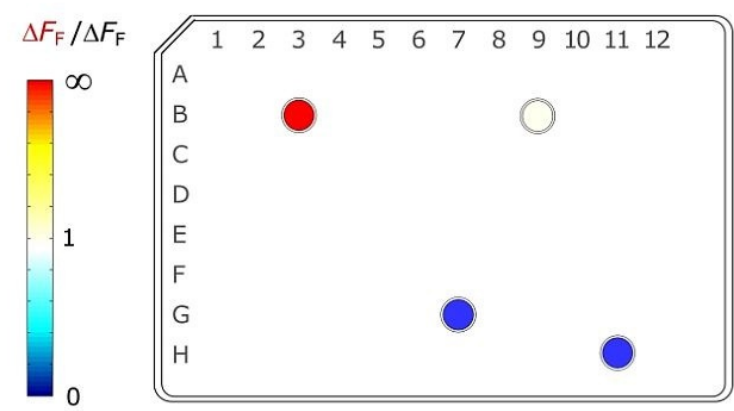

C
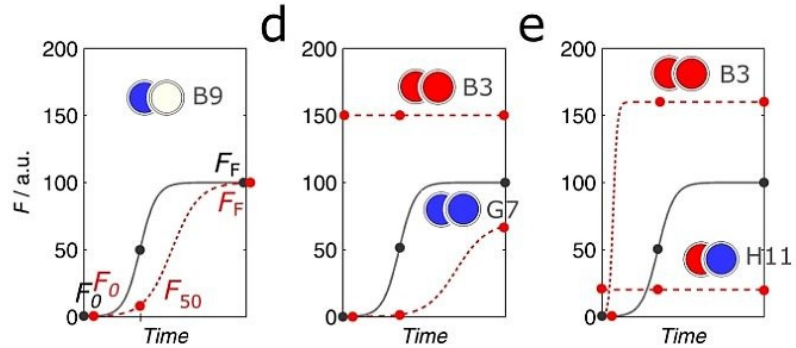

Figure 5. Possible readouts expected from the 3-point aggregation assay. a and b) Illustrative example of an arbitrary 96-well microplate with the assay output represented in terms of (a) the $\Delta F_{50} / \Delta F_{F}$ ratios and (b) the impact on the value of $\Delta F_{\mathrm{F}}$; different colors indicate different values of the readout as indicated in the colorbars. Cells B3, B9, G7 and H11 are positive hits identified in (a) and then further analyzed in (b). $c$ to e) The combinations of $\Delta F_{50}$ and $\Delta F_{F}$ values highlighted in (a) and in (b) apply to sigmoidal progress curves (lines) obtained in the absence (solid lines) and presence (dashed lines) of (c) kinetic inhibitors, (d) soluble protein stabilizers, and (e) fibril stabilizers/oligomer breakers. Horizontal dashed lines in (d) and (e) represent cases of undefined aggregation suppressors. 
to fibril stabilizers, oligomer breakers (both cases represented in Figure $5 \mathrm{e}$ ) or undefined aggregation suppressors (Figure $5 \mathrm{~d}$ ). To clarify this type of doubts, the initial hits-including the undefined results-have to undergo the next round of screenings based on dose-response, full progress curve analysis.

In addition to the conventional representation of $\alpha$ over time, amyloid aggregation curves are conveniently plotted in log-linear $\alpha /(1-\alpha)$ vs. time coordinates so as to detect deviations from the standard behavior expected by Equation (2). These deviations, which are dealt in detail elsewhere, ${ }^{[7]}$ are evidenced by non-linear trends arising after the initial lag phase. The relative importance of off-pathway aggregation generally increases as the curvature of the $\ln (\alpha /(1-\alpha))$ representation gets more pronounced. Therefore, distinguishing between similar signatures of aggregation modulators such as fibril stabilizers and oligomer breakers (Figures $3 \mathrm{c}, \mathrm{e}$ ), or monomer stabilizers and oligomer stabilizers (Figures $3 \mathrm{~d}, \mathrm{e}$ ) is possible by representing the reaction coordinates in the modified log-linear scale. Dose-response analyses of the assay measurables $t_{50}$, $v_{50}$ and $\Delta F_{F}$ are important to establish the potency of the selected compounds by means of the half-maximal inhibitory concentration $\left(\mathrm{IC}_{50}\right)$ and the half-maximal effective concentration $\left(E C_{50}\right)$ parameters. ${ }^{[4]}$ Yet, to answer the question on whether the right target has been reached, the relationships $\Delta F_{\mathrm{F}}, v_{50}$, and $t_{50}$ vs. protein concentration at constant compound concentration should also be looked in detail. In recent contributions we demonstrated that these scaling laws reflect the relative composition of monomers and soluble oligomers measured by direct methods. ${ }^{[9,43]}$ Specifically, a direct correspondence between the end-point amyloid signal and the initial concentration of monomer can be expected when no offpathway oligomers are present (Figure $6 \mathrm{a}$ ) or when their dissociation is much slower than the formation of amyloid fibrils (Figure $6 \mathrm{~b}$ ). This correspondence will not be observed if the oligomerization equilibrium is reversed by the gradual vanishing of protein monomers provoked by the amyloidogenic pathway (Figure $6 \mathrm{c}$ ). Examples of refractory and reversible offpathway oligomerization were observed during the aggregation of human insulin and ataxin-3, respectively. ${ }^{[9,43]}$

The oligomerization pathway can be further probed by analyzing the influence of protein concentration on the half-life coordinates. The scaling laws of $v_{50}$ (Figure $7 \mathrm{a}$ ) and $t_{50}$ (Figure $7 \mathrm{~b}$ ) show marked deviations from linearity in the presence of slowly dissociating oligomers, with unconventional scaling exponents $(\gamma)$ being admissible independently of the dominant step(s) of amyloid fibrillation (Figure $7 \mathrm{~b}$ ). Within the context of HTS secondary screenings, the analysis of protein concentration scaling laws in the presence and absence of test compounds is an effort worth making in order to discriminate which pathway is being affected and by which mechanisms.

Hit results found by indirect kinetic assays need to be validated by a combination of experimental methods capable of characterizing both quantitatively and qualitatively the target aggregates. The available options can be roughly categorized into spectroscopic, chromatographic, calorimetric, light scattering, microscopy and high-resolution techniques, which have
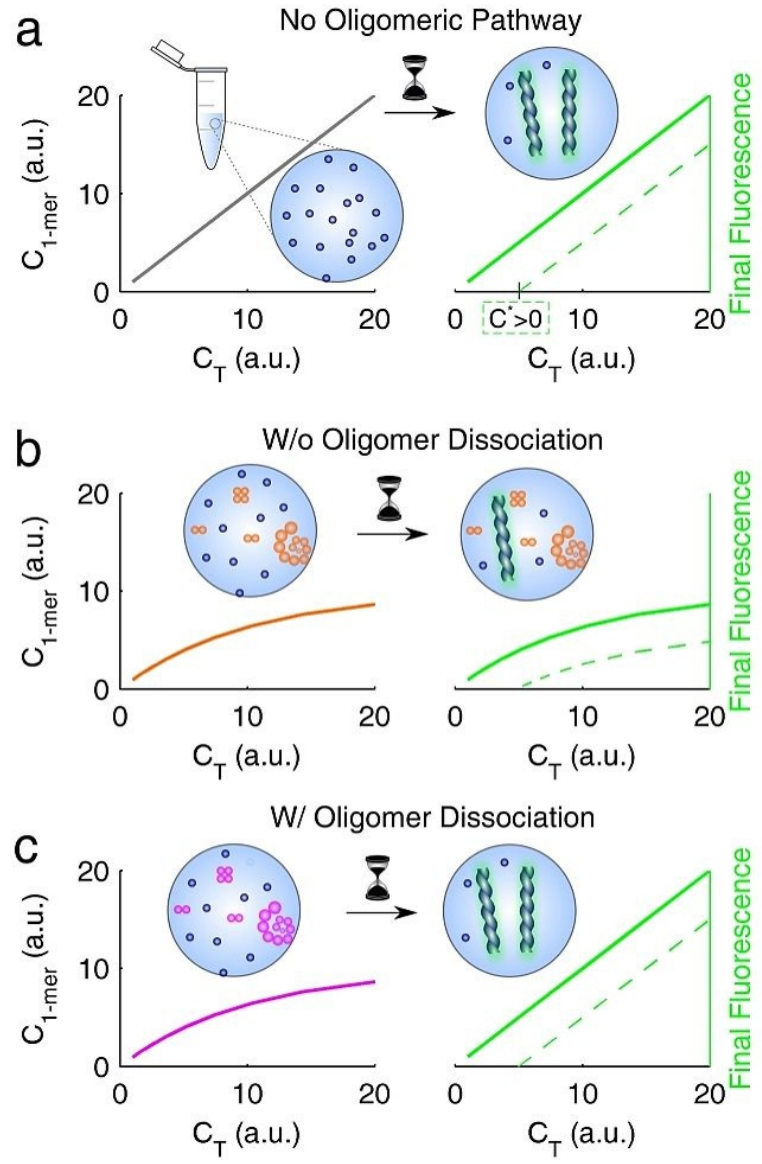

Figure 6. The scaling laws of final florescence with total protein concentration $\left(C_{T}\right)$ provide insights into off-pathway oligomerization-Figure reproduced under the terms of the CC BY 4.0 license from Silva et al.. ${ }^{[43]}$ The initial distributions of monomer and total protein (left side) influences the endpoint scaling laws (right side). The correspondence between the initial and final stages is direct if (a) no oligomerization pathway is present or (b) oligomerization is irreversible, and indirect if (c) oligomerization is fully reversible. Green lines describe cases of protein solubility values $C^{*}=0$ (solid lines) and $C^{*}>0$ (dashed lines).
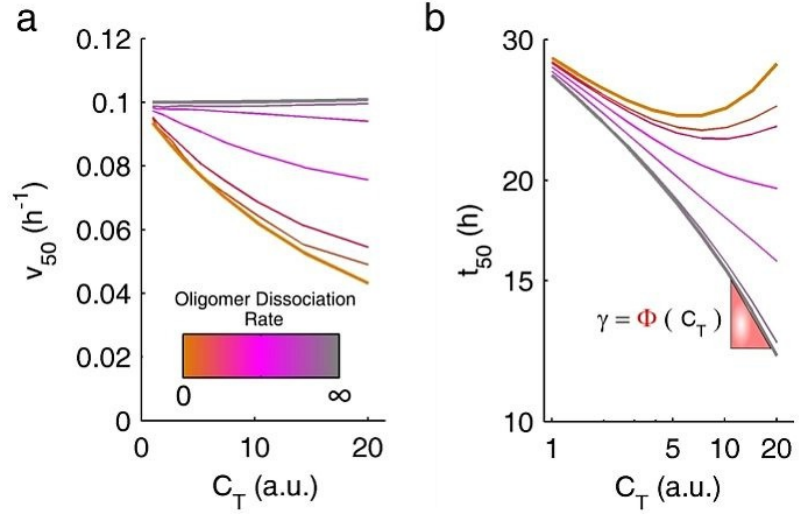

Figure 7. Highly diverse kinetic scaling laws are expected depending on the relative importance of off-pathway aggregation. The scaling laws of (a) $v_{50}$ and (b) $t_{50}$ change according to the initial degree of oligomerization and the rate at which off-pathway oligomers dissociate (color bar). The variability of the scaling exponents $(\gamma)$ cannot be explained if only on-pathway mechanisms are considered. Figure adapted under the terms of the CC BY $4.0 \mathrm{li}$ cense from Silva et al. ${ }^{[43]}$ assuming the cases of predominant secondary nucleation and hyperbolic initial distributions of monomers vs. total protein. 
been subject of updated reviews by for example, Poklar Ulrih, ${ }^{[45]}$ Pryor et al., ${ }^{[46]}$ and Renaud et al.. ${ }^{[4]}$ The application of these techniques may either confirm or deny the first indications resulting from the amyloid aggregation assay. Illustrating this, Wobst et al. ${ }^{[48]}$ employed circular dichroism (CD) to detect changes in the secondary structure of tau protein and to confirm that the green tea polyphenol (-)-epigallocatechin gallate (EGCG) prevents the formation of $\beta$-sheet-rich tau oligomers. On the other hand, Middleton et al. ${ }^{[49]}$ used isotope labelling and two-dimensional infrared spectroscopy to show how aggregation inhibitors may function by complex structural processes that are not detected by ThT fluorescence. Infrared nanospectroscopy has recently been used to characterize oligomeric and fibrillar species during amyloid formation of ataxin-3. ${ }^{[50]}$ Nuclear Magnetic Resonance (NMR) spectroscopy can provide high-resolution structural information as that used to unveil the ligand binding details of an anti-amyloidogenic compound (phthalocyanine tetrasulfonate) to $\alpha$-synuclein. Alternatively, NMR spectroscopy can be utilized to follow the formation of intermediate and off-pathway oligomers by periodically collecting the ${ }^{1} \mathrm{H}$ NMR spectra associated to monomer depletion. This has been done while studying the effect of $\mathrm{Cu}^{2+}$ on $\alpha$-synuclein aggregation, ${ }^{[51]}$ and to demonstrate the ability of EGCG to shift off-pathway aggregation of $A \beta 40 .{ }^{[52]}$ Solidstate $\mathrm{NMR}_{r}{ }^{[53]}$ together with X-ray microcrystallography, ${ }_{1}^{[54]} \mathrm{X}$-ray fiber diffraction ${ }^{[55]}$ and cryo-electron microscopy ${ }^{[56]}$ further enlarged the resolution limits of amyloid structures, ${ }^{[57]}$ and contributed to reveal morphological differences between amyloid fibrils generated in vitro or in the brain. ${ }^{[58]}$ With the recent advances in instrumentation, high intensity synchrotron sources, robotics/automation, speed of data processing, etc., some of the benchmark technologies are now being applied in de novo screenings of aggregation modulators. For example, inhibitors of human IAPP and A $\beta 40$ aggregation were identified by ion mobility spectrometry-mass spectrometry using ThT fluorescence monitoring as a control method to detect possible hydrophobic interactions that become lost in the gas phase. ${ }^{[59]}$ The scope of light scattering techniques is also expanding from proof-of-concept to high-throughput applications as in the cases of dynamic light scattering (DLS) for size distribution monitoring, ${ }^{[60]}$ and small angle X-ray scattering (SAXS) for characterizing the oligomeric equilibrium of non-aggregated samples. ${ }^{[61]}$ In another approach, a time-resolved fluorescence resonance energy transfer (FRET) assay was developed to screen $7.46 \times 10^{5}$ compounds and identify 56 hits that markedly inhibit $\alpha$-synuclein and several phenyl-benzoxazol compounds that promote $\alpha$-synuclein aggregation (proaggregators). ${ }^{[62]}$ Important for subsequent phases of hit to lead optimization, ${ }^{[5,18]}$ cell-based assays based on phenotypic and cytotoxicity readouts have also been used for compound screening purposes in the identification of IAAP aggregation inhibitors. ${ }^{[63]}$

\section{Conclusions and Perspectives}

Despite all the advances in the understanding of protein aggregation kinetics, the translation of this knowledge into new practical tools for drug discovery has been limited. We believe that this scenario is about to change on account of two reasons both connected with the key role played by soluble oligomers in the process of amyloid fibril formation. First, there is a growing interest in anti-oligomerization compounds as a possible therapeutic strategy to block the progression of neurodegenerative disorders and amyloidoses. Second, soluble oligomers, and particularly non-amyloidogenic off-pathway oligomers leave distinctive signatures in amyloid aggregation curves and scaling laws that help distinguishing which type of protein aggregates are being targeted. In the present work we reviewed the different mechanisms of action of known antiamyloid and anti-olgomerization compounds and showed how their effect can be identified in reaction progress curves. The systematization of this analysis led us to propose the 3-point aggregation assay for the primary screening of large libraries of chemical compounds based on ThT fluorescence readouts at the starting-point, (supposed) half-life and (supposed) endpoint instants of the reaction. We also reviewed the chemical kinetic tools available for the secondary screening and hit validation phases, which may involve the analysis of full progress curves represented in modified coordinates and the analysis of $\Delta F_{\mathrm{F}}, v_{50}$, and $t_{50}$ scaling laws.

\section{Acknowledgements}

This work was financed by (i) FEDER-Fundo Europeu de Desenvolvimento Regional funds through the COMPETE 2020-Operacional Programme for Competitiveness and Internationalisation (POCI), Portugal 2020, and by Portuguese funds through FCTFundação para a Ciência e a Tecnologia/Ministério da Ciência, Tecnologia e Ensino Superior in the framework of the projects POCI-01-0145-FEDER-031173 (PTDC/BIA-BFS/31173/2017), POCI01-0145-FEDER-007274, POCI-01-0145-FEDER-031323 ("Institute for Research and Innovation in Health Sciences") and POCI-010145-FEDER-006939 (Laboratory for Process Engineering, Environment, Biotechnology and Energy-UID/EQU/00511/2013), and by (ii) FEDER through Norte Portugal Regional Operational Programme (NORTE 2020), under the PORTUGAL 2020 Partnership Agreement in the framework of Project Norte-01-0145FEDER-000008 and by national funds (PIDDAC) through FCT project "LEPABE-2-ECO-INNOVATION"-NORTE-01-0145-FEDER000005.

\section{Conflict of interest}

The authors declare no conflict of interest.

Keywords: amyloid diseases · drug discovery $\cdot$ nucleation protein aggregation $\cdot$ soluble oligomers

[1] H.-P. Shih, X. Zhang, A. M. Aronov, Nat. Rev. Drug Discovery 2018, 17, 19.

[2] a) D. M. Walsh, D. J. Selkoe, Nat. Rev. Neurosci. 2016, 17, 251; b) M. L. Choi, S. Gandhi, FEBS J. 2018, 285, 3631.

[3] D. J. Selkoe, Ann. Neurol. 2013, 74, 328. 
[4] a) J. Sevigny, P. Chiao, T. Bussière, P. H. Weinreb, L. Williams, M. Maier, R. Dunstan, S. Salloway, T. Chen, Y. Ling, Nature 2016, 537, 50; b) D. B. Schenk, M. Koller, D. K. Ness, S. G. Griffith, M. Grundman, W. Zago, J. Soto, G. Atiee, S. Ostrowitzki, G. G. Kinney, Mov. Disord. 2017, 32, 211

[5] R. Macarron, M. N. Banks, D. Bojanic, D. J. Burns, D. A. Cirovic, T. Garyantes, D. V. Green, R. P. Hertzberg, W. P. Janzen, J. W. Paslay, Nat. Rev. Drug Discovery 2011, 10, 188.

[6] A. Villar-Piqué, M. Schmitz, N. Candelise, S. Ventura, F. Llorens, I. Zerr, Mol. Neurobiol. 2018, 1.

[7] R. Crespo, E. Villar-Alvarez, P. Taboada, F. A. Rocha, A. M. Damas, P. M. Martins, J. Biol. Chem. 2016, 291, 2018.

[8] R. Crespo, E. Villar-Alvarez, P. Taboada, F. A. Rocha, A. M. Damas, P. M. Martins, J. Phys. Chem. B 2017, 121, 2288.

[9] A. Silva, B. Almeida, J. S. Fraga, P. Taboada, P. M. Martins, S. Macedo-Ribeiro, Angew. Chem. Int. Ed. 2017, 56, 14042; Angew. Chem. 2017, 129, 14230.

[10] G. Meisl, J. B. Kirkegaard, P. Arosio, T. C. Michaels, M. Vendruscolo, C. M. Dobson, S. Linse, T. P. Knowles, Nat. Protoc. 2016, 11, 252.

[11] a) N. Cremades, C. M. Dobson, Neurobiol. Dis. 2018, 109, 178; b) J. Pujols, S. Peña-Díaz, D. F. Lázaro, F. Peccati, F. Pinheiro, D. González, A Carija, S. Navarro, M. Conde-Giménez, J. García, S. Guardiola, E. Giralt, X. Salvatella, J. Sancho, M. Sodupe, T. F. Outeiro, E. Dalfó, S. Ventura, Proc. Natl. Acad. Sci. USA 2018, 115, 10481

[12] R. Crespo, F. A. Rocha, A. M. Damas, P. M. Martins, J. Biol. Chem. 2012 287, 30585

[13] L. Bentea, M. A. Watzky, R. G. Finke, J. Phys. Chem. C 2017, 121, 5302.

[14] J. N. Buxbaum, R. P. Linke, J. Mol. Biol. 2012, 421, 142.

[15] I. Dolado, J. Nieto, M. J. M. Saraiva, G. Arsequell, G. Valencia, A. Planas, J. Comb. Chem. 2005, 7, 246.

[16] D. J. Lindberg, A. Wenger, E. Sundin, E. Wesén, F. Westerlund, E. K. Esbjörner, Biochemistry 2017, 56, 2170.

[17] a) C. Aldrich, C. Bertozzi, G. I. Georg, L. Kiessling, C. Lindsley, D. Liotta, K. M. Merz, A. Schepartz, S. Wang, ACS Cent. Sci. 2017, 3, 143; b) N Thorne, D. S. Auld, J. Inglese, Curr. Opin. Chem. Biol. 2010, 14, 315.

[18] A. Roy, High-Throughput 2018, 7, 4

[19] D. Kashchiev, R. Cabriolu, S. Auer, J. Am. Chem. Soc. 2013, 135, 1531.

[20] K. G. Malmos, L. M. Blancas-Mejia, B. Weber, J. Buchner, M. Ramirez-Alvarado, H. Naiki, D. Otzen, Amyloid 2017, $24,1$.

[21] J.-H. Zhang, T. D. Chung, K. R. Oldenburg, J. Biomol. Screening 1999, 4, 67.

[22] L. Giehm, D. E. Otzen, Anal. Biochem. 2010, 400, 270.

[23] J. Howitt, A. F. Hill, J. Biol. Chem. 2016, 291, 26589.

[24] a) M. Grey, C. J. Dunning, R. Gaspar, C. Grey, P. Brundin, E. Sparr, S. Linse, J. Biol. Chem. 2015, 290, 2969; b) R. Gaspar, J. Pallbo, U. Weininger, S Linse, E. Sparr, Biochim. Biophys. Acta Proteins Proteomics 2018, 1866 1062

[25] A. J. Doig, P. Derreumaux, Curr. Opin. Struct. Biol. 2015, 30, 50.

[26] Y. S. Eisele, C. Monteiro, C. Fearns, S. E. Encalada, R. L. Wiseman, E. T. Powers, J. W. Kelly, Nat. Rev. Drug Discovery 2015, 14, 759.

[27] P. Velander, L. Wu, F. Henderson, S. Zhang, D. R. Bevan, B. Xu, Biochem Pharmacol. 2017, 139, 40.

[28] J. A. Loureiro, R. Crespo, H. Börner, P. M. Martins, F. A. Rocha, M. Coelho, M. C. Pereira, S. Rocha, J. Mater. Chem. B 2014, 2, 2259.

[29] S. Sinha, D. H. J. Lopes, Z. Du, E. S. Pang, A. Shanmugam, A. Lomakin, P. Talbiersky, A. Tennstaedt, K. McDaniel, R. Bakshi, P.-Y. Kuo, M. Ehrmann G. B. Benedek, J. A. Loo, F.-G. Klärner, T. Schrader, C. Wang, G. Bitan, J. Am. Chem. Soc. 2011, 133, 16958.

[30] T. Schrader, G. Bitan, F.-G. Klärner, Chem. Commun. 2016, 52, 11318.

[31] W.-J. Du, J.-J. Guo, M.-T. Gao, S.-Q. Hu, X.-Y. Dong, Y.-F. Han, F.-F. Liu, S Jiang, Y. Sun, Sci. Rep. 2015, 5, 7992.

[32] T.-P. V. Huynh, D. M. Holtzman, Trends Neurosci. 2018, 41, 483.

[33] L. Jiang, C. Liu, D. Leibly, M. Landau, M. Zhao, M. P. Hughes, D. S. Eisenberg, elife 2013, 2, e00857.

[34] L.-M. Yan, A. Velkova, M. Tatarek-Nossol, G. Rammes, A. Sibaev, E. Andreetto, M. Kracklauer, M. Bakou, E. Malideli, B. Göke, J. Schirra, M. Storr A. Kapurniotu, Angew. Chem. Int. Ed. 2013, 52, 10378; Angew. Chem. 2013, 125, 10569

[35] T. Coelho, G. Merlini, C. E. Bulawa, J. A. Fleming, D. P. Judge, J. W. Kelly, M. S. Maurer, V. Planté-Bordeneuve, R. Labaudiniere, R. Mundayat, Neurol. Ther. 2016, 5, 1.
[36] J. L. Berk, O. B. Suhr, L. Obici, Y. Sekijima, S. R. Zeldenrust, T. Yamashita, M. A. Heneghan, P. D. Gorevic, W. J. Litchy, J. F. Wiesman, JAMA J. Am Med. Assoc. 2013, 310, 2658.

[37] R. Sant'Anna, P. Gallego, L. Z. Robinson, A. Pereira-Henriques, N. Ferreira, F. Pinheiro, S. Esperante, I. Pallares, O. Huertas, M. R. Almeida, N. Reix ach, R. Insa, A. Velazquez-Campoy, D. Reverter, N. Reig, S. Ventura, Nat Commun. 2016, 7, 10787.

[38] H. Amijee, C. Bate, A. Williams, J. Virdee, R. Jeggo, D. Spanswick, D. I. C. Scopes, J. M. Treherne, S. Mazzitelli, R. Chawner, C. E. Eyers, A. J. Doig Biochemistry 2012, 51, 8338.

[39] a) A. Campos-Ramírez, M. Márquez, L. Quintanar, L. F. Rojas-Ochoa, Biophys. Chem. 2017, 228, 98; b) M. Adachi, M. Noji, M. So, K. Sasahara, J. Kardos, H. Naiki, Y. Goto, J. Biol. Chem. 2018, 293, 14775.

[40] a) Y. Goto, M. Adachi, H. Muta, M. So, Biophys. Rev. 2018, 10, 493; b) C. Ferreira, F. A. Rocha, A. M. Damas, P. M. Martins, Cryst. Growth Des. 2016 16,4285 .

[41] J. Pronchik, X. He, J. T. Giurleo, D. S. Talaga, J. Am. Chem. Soc. 2010, 132, 9797.

[42] P. M. Martins, Prion 2013, 7, 136

[43] A. Silva, Z. Sárkány, J. Fraga, P. Taboada, S. Macedo-Ribeiro, P. Martins, Biomolecules 2018, 8, 108 .

[44] B. Y. Feng, B. H. Toyama, H. Wille, D. W. Colby, S. R. Collins, B. C. H. May, S. B. Prusiner, J. Weissman, B. K. Shoichet, Nat. Chem. Biol. 2008, 4, 197.

[45] N. P. Ulrih, Crit. Rev. Food Sci. Nutr. 2017, 57, 2144

[46] N. E. Pryor, M. A. Moss, C. N. Hestekin, Int. J. Mol. Sci. 2012, 13, 3038

[47] J.-P. Renaud, C.-w. Chung, U. H. Danielson, U. Egner, M. Hennig, R. E. Hubbard, H. Nar, Nat. Rev. Drug Discovery 2016, 15, 679.

[48] H. J. Wobst, A. Sharma, M. I. Diamond, E. E. Wanker, J. Bieschke, FEBS Lett. 2015, 589, 77.

[49] C. T. Middleton, P. Marek, P. Cao, C.-C. Chiu, S. Singh, A. M. Woys, J. J. de Pablo, D. P. Raleigh, M. T. Zanni, Nat. Chem. 2012, 4, 355.

[50] F. S. Ruggeri, G. Longo, S. Faggiano, E. Lipiec, A. Pastore, G. Dietler, Nat. Commun. 2015, 6, 7831

[51] A. Villar-Piqué, T. L. da Fonseca, R. Sant'Anna, É. M. Szegö, L. Fonseca-Ornelas, R. Pinho, A. Carija, E. Gerhardt, C. Masaracchia, E. A. Gonzalez, G. Rossetti, P. Carloni, C. O. Fernández, D. Foguel, I. Milosevic, M. Zweckstetter, S. Ventura, T. F. Outeiro, Proc. Natl. Acad. Sci. USA 2016, 113, E6506.

[52] J. Wang, T. Yamamoto, J. Bai, S. J. Cox, K. J. Korshavn, M. Monette, A. Ramamoorthy, Chem. Commun. 2018, 54, 2000.

[53] R. Tycko, Biophys. J. 2018, 114, 185a.

[54] R. Riek, D. S. Eisenberg, Nature 2016, 539, 227.

[55] Y. K. Al-Hilaly, S. J. Pollack, D. M. Vadukul, F. Citossi, J. E. Rickard, M. Simpson, J. M. D. Storey, C. R. Harrington, C. M. Wischik, L. C. Serpell, J. Mol. Biol. 2017, 429, 3650

[56] B. Li, P. Ge, K. A. Murray, P. Sheth, M. Zhang, G. Nair, M. R. Sawaya, W. S Shin, D. R. Boyer, S. Ye, D. S. Eisenberg, Z. H. Zhou, L. Jiang, Nat Commun. 2018, 9, 3609

[57] F. Chiti, C. M. Dobson, Annu. Rev. Biochem. 2017, 86, 27.

[58] a) J. L. Guo, V. M. Y. Lee, Nat. Med. 2014, 20, 130; b) W. Qiang, W.-M. Yau, J.-X. Lu, J. Collinge, R. Tycko, Nature 2017, 541, 217.

[59] L. M. Young, J. C. Saunders, R. A. Mahood, C. H. Revill, R. J. Foster, L.-H. Tu, D. P. Raleigh, S. E. Radford, A. E. Ashcroft, Nat. Chem. 2015, 7, 73

[60] P. Nedumpully-Govindan, A. Kakinen, E. H. Pilkington, T. P. Davis, P. C. Ke, F. Ding, Sci. Rep. 2016, 6, 19463.

[61] M. A. Graewert, D. Franke, C. M. Jeffries, C. E. Blanchet, D. Ruskule, K. Kuhle, A. Flieger, B. Schäfer, B. Tartsch, R. Meijers, D. I. Svergun, Sci. Rep. 2015, 5, 10734

[62] M. Kurnik, C. Sahin, C. B. Andersen, N. Lorenzen, L. Giehm, H. Mohammad-Beigi, C. M. Jessen, J. S. Pedersen, G. Christiansen, S. V. Petersen, R. Staal, G. Krishnamurthy, K. Pitts, P. H. Reinhart, F. A. A. Mulder, S. Mente W. D. Hirst, D. E. Otzen, Cell Chem. Biol. 2018, 25, 1389.

[63] J. C. Saunders, L. M. Young, R. A. Mahood, M. P. Jackson, C. H. Revill, R. J. Foster, D. A. Smith, A. E. Ashcroft, D. J. Brockwell, S. E. Radford, Nat. Chem. Biol. 2016, 12, 94.

Manuscript received: November 20, 2018

Revised manuscript received: January 11, 2019

Accepted manuscript online: January 15, 2019

Version of record online: February 1, 2019 